\title{
Wave instabilities of a collisionless plasma in fluid approximation
}

\author{
Namig S. Dzhalilov ${ }^{1,2,3, *}$, Vladimir D. Kuznetsov ${ }^{2, * *}$, and Jürgen Staude ${ }^{1, * * *}$. \\ ${ }^{1}$ Astrophysikalisches Institut Potsdam (AIP), An der Sternwarte 16, D-14482 Potsdam, Germany \\ ${ }^{2}$ Pushkov Institute of Terrestrial Magnetism, Ionosphere and Radio Wave Propagation of the Russian Academy \\ of Sciences, (IZMIRAN), Troitsk City, Moscow Region, 142190 Russia \\ ${ }^{3}$ Shamakhy Astrophysical Observatory of the Azerbaijan Academy of Sciences (ShAO), Baku Az-1000, Azer- \\ baijan
}

Received 30 July 2010, accepted 10 November 2010

Published online xx December 2010

Key words magnetohydrodynamics (MHD), instabilities, plasmas, waves, Sun: corona, solar wind.

Wave properties and instabilities in a magnetized, anisotropic, collisionless, rarefied hot plasma in fluid approximation are studied, using the 16-moments set of the transport equations obtained from the Vlasov equations. These equations differ from the CGL-MHD fluid model (single fluid equations by Chew, Goldberger, and Low [9,5]) by including two anisotropic heat flux evolution equations, where the fluxes invalidate the double polytropic CGL laws. We derived the general dispersion relation for linear compressible wave modes. Besides the classic incompressible fire hose modes there appear four types of compressible wave modes: two fast and slow mirror modes - strongly modified compared to the CGL model - and two thermal modes. In the presence of initial heat fluxes along the magnetic field the wave properties become different for the waves running forward and backward with respect to the magnetic field. The well known discrepancies between the results of the CGL-MHD fluid model and the kinetic theory are now removed: i) The mirror slow mode instability criterion is now the same as that in the kinetic theory. ii) Similarly, in kinetic studies there appear two kinds of fire hose instabilities - incompressible and compressible ones. These two instabilities can arise for the same plasma parameters, and the instability of the new compressible oblique fire hose modes can become dominant. The compressible fire hose instability is the result of the resonance coupling of three retrograde modes - two thermal modes and a fast mirror mode. The results can be applied to the theory of solar and stellar coronal and wind models.

Copyright line will be provided by the publisher

\section{Introduction}

Frequent particle collisions turn the plasma distribution function into an isotropic one, and thus the thermal pressure is isotropic as well. If collisions rarely occur the presence of a magnetic field will maintain a "nonmixed" state of the energies of the longitudinal and transverse motions of particles. Thus, the transverse and longitudinal kinetic particle temperatures will differ from each other, $T_{\perp} \neq T_{\|}$. Typical examples of such plasmas are the coronal and solar wind plasmas which are very anisotropic and inhomogeneous, in cross-field direction in particular [2]. For the observational motivation of our modeling see the references in our previous paper [11]. So, due to the anisotropy of the kinetic temperatures of the particles (especially protons and heavy ions) the corresponding partial pressures become anisotropic in this way. This makes the total thermal pressure anisotropic too, $p_{\perp} \neq p_{\|}$.

In the corona the electron and ion gyroradii $r_{B}$ and gyrotimes $\tau_{B}$ become smaller than any of the particle collisional mean free paths and times and smaller than any of the typical scales of variations of macroscopic thermodynamical quantities. Thus, the condition of a strongly magnetized plasma is well satisfied. That means, particles gyrating around the magnetic field lines are localized across the field at a distance of the Larmor radius which plays the role of a free path length of particles. Thus, the dynamical motion of a collisionless plasma with characteristic scales of $L \gg r_{B}$ and $\tau \gg \tau_{B}$ behaves across the magnetic field as a fluid. However, under

\footnotetext{
* E-mail: NamigD@mail.ru

** E-mail: KVD@izmiran.ru

*** Corresponding author: E-mail: jstaude@aip.de, Phone \& Fax: +493327 43596
} 
such circumstances a traditional hydrodynamical description of the plasma is hardly possible [41]. The isotropic MHD equations are applicable only if the plasma is collision-dominated and the distribution functions are close to Maxwellian's. In the opposite limiting case, when the plasma is collisionless at all, the local distribution function strongly differs from the Maxwell function. To describe the plasma in the fluid approximation in this case usually the single fluid CGL-MHD equations by Chew et al. [9,5] are applied. Instead of the energy equation of the isotropic MHD these equations include double-polytropic laws in the form $p_{\perp} / \rho B=$ const and $p_{\|} B^{2} / \rho^{3}=$ const. Many studies of wave instability problems are based on these equations. Similar to the low-frequency kinetic considerations there appear two kinds of instabilities: incompressible fire hose and compressible mirror instabilities [57, 8, 48, 6, 17, 13]. In comparison to the results based on the kinetic theory the CGL equations provide the correct instability criterion for the classic fire hose instability. However, there exists a discrepancy in the criterion for the slow-mode mirror instability. Moreover, there appear basic differences between the nonlinear stages of these instabilities compared with the kinetic results. It has been shown [21, 22] by hybrid kinetic simulations that a new type of fire hose instability may arise for oblique propagation due to the proton temperature anisotropy. Unlike the classical fire hose instability this instability is compressible and has a maximum growth rate at oblique propagation. The growth rate of the new instability is comparable to (or in some parameters ranges even larger than) the maximum of the standard fire hose instability growth rate. Both fire hose instabilities may occur at the same time for the same plasma parameters. A similar second type of fire hose instability driven by the electron temperature anisotropy has also been found [24,47,37]. The CGL theory cannot give an analogy of this second type of fire hose instability.

To remove such discrepancies in the CGL-MHD model, generalized polytropic laws were used [1, 19, 58] introducing some artificial polytropic indices such as $p_{\perp} / \rho B^{\gamma_{1}-1}=$ const and $p_{\|} B^{\gamma_{2}-1} / \rho^{\gamma_{2}}=$ const. With a suitable choice of these free polytropic indices, $\gamma_{1}$ and $\gamma_{2}$, it is in principle possible to remove some of the discrepancies. However, the origin of these new indices is not clear as they do not follow directly from the kinetic equations when the fluid equations are derived. Later it has been shown that in the experimental data and in the particle simulations these two adiabatic invariants become invalid for realistic plasmas [40,49]. The conservation of the two CGL adiabatic invariants in an ideal collisionless plasma leads to a strong pressure anisotropy $p_{\perp}<p_{\|}$ which is much larger than the observed values [16,23]. However, due to non-ideal effects such as heat flux these invariants are broken [20], and this leads to properties which are quiet different from those predicted by the CGL equations. Deriving the CGL-MHD equations the third moments of the distribution function, hence the heat fluxes have been ignored without any proof [5], which is the main shortage of these equations.

In the present paper we study the linear wave instability problem on the base of more correct equations the 16-moments transport equations, which are derived from the Vlasov collisionless magnetized plasma kinetic equations by the fast gyromotion ordering technique [45, 46, 50]. These equations include additionally two dynamic evolution equations of the heat fluxes and no polytropic laws are possible. This allows us to resolve the main discrepancies of the CGL fluid theory. We consider the wave peculiarities which can appear in the anisotropic compressible plasma. We have already considered the incompressible wave instability on the base of these equations [11]. In Section 2 we formulate the basic equations, which are the integrated moment equations of the kinetic Vlasov equations. In Section 3 the linear compressible wave equation and the general dispersion relation are derived. To compare the results with the CGL-MHD theory the CGL dispersion equation is deduced from the new dispersion equation as a special case in Section 4. The solutions and analysis of the new dispersion equation are the topic of Section 5. In Section 6 we show that similar to the the kinetic theory the existence of two kinds of fire hose instabilities is possible in the fluid approximation too. The mass density fluctuations due to compressible wave modes and their instability is discussed in the Section 7. A discussion and some conclusions are presented in Section 8.

\section{Basic equations}

A plasma is described by the system of kinetic equations for the distribution functions of the particles and the Maxwell equations for the electromagnetic field. Due to the complexity of the kinetic equations the large-scale behavior of the plasma is usually described by deducing the equations for the integrated moments of the distribution function, and these equations are referred to as the hydrodynamical or the transport equations. The set of usual MHD equations is one variant of such equations valid for the collision-dominated isotropic plasma. For 
the description of a collisionless anisotropic plasma the 16-moments set of equations may be used which is more complete including the evolution of heat fluxes. This set of equations has been used by many authors in different theoretical approaches, especially for modeling the ionospheric plasma [45, 46] and the solar wind [10 44, 36, 38]. A more correct and compact form of these single-fluid transport equations for the anisotropic plasma in the presence of gravity $g$ but without magnetic diffusivity under the conditions $r_{B} \ll V \tau$ and $r_{B} \ll v_{T} \tau$ has been derived [46]; see [45, 50]. These equations are given as follows

$$
\begin{aligned}
& \frac{d \rho}{d t}+\rho \operatorname{div} \vec{v}=0 \\
& \rho \frac{d \vec{v}}{d t}+\nabla\left(p_{\perp}+\frac{B^{2}}{8 \pi}\right)-\frac{1}{4 \pi}(\vec{B} \cdot \nabla) \vec{B}=\rho \vec{g}+\left(p_{\perp}-p_{\|}\right)[\vec{h} \operatorname{div} \vec{h}+(\vec{h} \cdot \nabla) \vec{h}]+\vec{h}(\vec{h} \cdot \nabla)\left(p_{\perp}-p_{\|}\right), \\
& \frac{d}{d t} \frac{p_{\|} B^{2}}{\rho^{3}}=-\frac{B^{2}}{\rho^{3}}\left[B(\vec{h} \cdot \nabla)\left(\frac{S_{\|}}{B}\right)+\frac{2 S_{\perp}}{B}(\vec{h} \cdot \nabla) B\right] \\
& \frac{d}{d t} \frac{p_{\perp}}{\rho B}=-\frac{B}{\rho}(\vec{h} \cdot \nabla)\left(\frac{S_{\perp}}{B^{2}}\right) \\
& \frac{d}{d t} \frac{S_{\|} B^{3}}{\rho^{4}}=-j \frac{3 p_{\|} B^{3}}{\rho^{4}}(\vec{h} \cdot \nabla)\left(\frac{p_{\|}}{\rho}\right) \\
& \frac{d}{d t} \frac{S_{\perp}}{\rho^{2}}=-j \frac{p_{\|}}{\rho^{2}}\left[(\vec{h} \cdot \nabla)\left(\frac{p_{\perp}}{\rho}\right)+\frac{p_{\perp}}{\rho} \frac{p_{\perp}-p_{\|}}{p_{\|} B}(\vec{h} \cdot \nabla) B\right] \\
& \frac{d \vec{B}}{d t}+\vec{B} \operatorname{div} \vec{v}-(\vec{B} \cdot \nabla) \vec{v}=0, \\
& \operatorname{div} \vec{B}=0,
\end{aligned}
$$

where $\nabla=\nabla_{\|}+\nabla_{\perp}, \nabla_{\|}=\vec{h}(\vec{h} \cdot \nabla)$, and $\frac{d}{d t}=\frac{\partial}{\partial t}+(\vec{v} \cdot \nabla), \vec{v}=\overrightarrow{v_{\|}}+\overrightarrow{v_{\perp}}, \vec{h}=\frac{\vec{B}}{B}$. Here $S_{\|}$and $S_{\perp}$ are the heat fluxes along the magnetic field by parallel and perpendicular thermal kinetic motions. If the heat fluxes are neglected, $S_{\perp}=0$ and $S_{\|}=0$, we obtain the equations describing the laws of the change of longitudinal and transverse thermal energy along the trajectories of the plasma (the left-hand parts of Eqs. (3 4 4 ). These so-called "double-adiabatic" parities and Eqs. (1), (2), (7), and (8) form a closed system of equations, the CGL (Chew-Goldberger-Low) equations, see the pioneering work by Chew et al. (1956). However, the CGL-equations can result in unsatisfactory heat flux evolution Eqs. (5 6). This is because deducing the CGL equations the third moments of the distribution function, hence the heat fluxes, have been lost without any proof [9] 5]. The equations following from the 16-moments set in our case, Eqs. (1 8), consider the heat fluxes, they are more complete, and the CGL equations do not follow from these equations as a special case. One should compare the final results in the limits $S_{\perp} \rightarrow 0$ and $S_{\|} \rightarrow 0$ with the results based on the CGL equations, deduced by many authors [29,5], 33].

To consider the CGL equations separately, we introduce on the right-hand sides of the heat flux evaluation Eqs. (5-6) the parameter $j$. To reach the exact CGL equations we should take $S_{\|}=S_{\perp}=0$ and put $j=0$. In the general non-CGL case $S_{\|} \neq 0, S_{\perp} \neq 0$, and $j \equiv 1$.

\section{Wave equations}

For simplicity we will now assume, that the basic initial equilibrium state of the spatially non-limited plasma is homogeneous, $g=0$, and the following quantities are constant: $v_{0}, \rho_{0}, p_{\perp 0}, p_{\| 0}, B_{0}, S_{\perp 0}$, and $S_{\| 0}$. Eqs. (1) 8) will automatically satisfy such an equilibrium state with non-zero initial heat fluxes. We will consider small linear perturbations of all physical variables, e.g. for pressure in the form $p=p_{0}+p^{\prime}(r, t)$. Let $p^{\prime}(r, t) \sim$ $\exp i(\vec{k} \cdot \vec{r}-\omega t)$, where $\omega=\omega_{0}+\left(\overrightarrow{v_{0}} \cdot \vec{k}\right)$ is the wave frequency observed in the moving frame of the fluid, and 
$k$ is the wave number of the fluctuations. For the perturbations we obtain the equations

$$
\begin{aligned}
& \omega \rho^{\prime}-\rho_{0}(\vec{k} \cdot \vec{v})=0, \\
& \omega \rho_{0} \vec{v}-\vec{k}\left(p_{\perp}^{\prime}+\frac{\overrightarrow{B_{0}} \cdot \overrightarrow{B^{\prime}}}{4 \pi}\right)+k_{\|} \frac{B_{0}}{4 \pi} \overrightarrow{B^{\prime}}-\Delta\left[\overrightarrow{h_{0}}\left(\vec{k} \cdot \overrightarrow{h^{\prime}}\right)+k_{\|} \overrightarrow{h^{\prime}}\right]-k_{\|} \overrightarrow{h_{0}}\left(p_{\|}^{\prime}-p_{\perp}^{\prime}\right)=0, \\
& \omega \overrightarrow{B^{\prime}}-\overrightarrow{B_{0}}(\vec{k} \cdot \vec{v})+\left(\vec{k} \cdot \overrightarrow{B_{0}}\right) \vec{v}=0, \\
& \left(\vec{k} \cdot \overrightarrow{B^{\prime}}\right)=0, \\
& a_{0} \frac{p_{\perp}^{\prime}}{p_{\perp 0}}=a_{1} \frac{B^{\prime}}{B_{0}}+a_{2} \frac{\rho^{\prime}}{\rho_{0}}, b_{0} \frac{p_{\|}^{\prime}}{p_{\| 0}}=b_{1} \frac{B^{\prime}}{B_{0}}+b_{2} \frac{\rho^{\prime}}{\rho_{0}} .
\end{aligned}
$$

In deriving these equations, we have expressed the fluctuations of the thermal fluxes as

$$
\begin{aligned}
S_{\perp}^{\prime} & =j \frac{k_{\|} p_{\| 0} p_{\perp 0}}{\omega \rho_{0}}\left[\frac{p_{\perp}^{\prime}}{p_{\perp 0}}-\frac{\rho^{\prime}}{\rho_{0}}-\frac{\Delta}{p_{\| 0}} \frac{B^{\prime}}{B_{0}}\right]+2 S_{\perp 0} \frac{\rho^{\prime}}{\rho_{0}} \\
S_{\|}^{\prime} & =j \frac{3 p_{\| 0}^{2} k_{\|}}{\rho_{0} \omega}\left(\frac{p_{\|}^{\prime}}{p_{\| 0}}-\frac{\rho^{\prime}}{\rho_{0}}\right)-S_{\| 0}\left(3 \frac{B^{\prime}}{B_{0}}-4 \frac{\rho^{\prime}}{\rho_{0}}\right) .
\end{aligned}
$$

Here $\Delta=p_{\| 0}-p_{\perp 0}, \overrightarrow{h_{0}}=\overrightarrow{B_{0}} / B_{0}, k_{\|}=\left(\overrightarrow{h_{0}} \cdot \vec{k}\right)=k \cos \theta$. The indices $\|$ and $\perp$ correspond to the values of the parameters along and across the magnetic field, respectively. Even if we insert in Eqs. 114 15 $S_{\| 0}=S_{\perp 0}=0$, the perturbations of these functions will never become zero: $S_{\|}^{\prime} \neq 0, S_{\perp}^{\prime} \neq 0$. That means, using the 16-moments equations we should get more reliable results on the wave properties in an anisotropic plasma than with the CGL equations based on the 13-moments equations.

Strongly speaking, the initial heat fluxes are defined at the kinetic level as the third moments of the particle distribution function. In the presence of an external magnetic field the components of this flux are defined by the steady solutions of the kinetic equation. However, we should use here some appropriate estimate as a parameter.

The initial collisionless heat flux functions $S_{\| 0}$ and $S_{\perp 0}$ should be estimated by taking the thermal energy density of the electrons multiplied by the particle stream speed along the magnetic field $u_{0}$. For example, for the solar wind plasma we can write $S_{\| 0} \approx \frac{3}{2} n_{\mathrm{e}} k_{B} T_{\|} u_{0} \delta=\frac{3}{4} \delta u_{0} p_{\|}$. Hollweg [25, 26] has given some estimates of the correction parameter $\delta$ ( $\alpha$ in his papers) assuming various realistic shapes of the electron distribution function and checking the results for agreement with space observations. $\delta$ depends on the magnetic field. In the range $B=0.1-100 \mathrm{G}\left(1 \mathrm{G}=10^{-4} \mathrm{~T}\right)$ we have $\delta \approx 4-0.1$. In the same way $S_{\perp 0} \approx \frac{3}{4} \delta u_{0} p_{\perp}$.

Let us introduce dimensionless parameters (in the further text indices ' 0 ' of physical parameters will be omitted for simplicity):

$$
\begin{aligned}
& \alpha=\frac{p_{\perp}}{p_{\|}}, \bar{\alpha}=1-\alpha, c_{\|}^{2}=\frac{p_{\|}}{\rho}, \beta=\frac{B^{2}}{4 \pi p_{\|}}=\frac{v_{A}^{2}}{c_{\|}^{2}}, \eta=\frac{c_{\|} k_{\|}}{\omega}=\frac{c_{\|} k}{\omega} \cos \theta, \\
& \bar{S}_{\|}=\frac{S_{\|}}{p_{\|} c_{\|}}, \overline{S_{\perp}}=\frac{S_{\perp}}{p_{\perp} c_{\|}}, \bar{S}=\alpha \overline{S_{\perp}}-2 \bar{S}_{\|}, l_{1}=\cos ^{2} \theta, l_{2}=\sin ^{2} \theta .
\end{aligned}
$$

Note that $\beta$ is defined here inversely proportional to the often used plasma beta. Having in mind the approximate estimates of the initial heat fluxes we may introduce the dimensionless parameter $\gamma=(3 / 4) \delta u_{0} / c_{\|}$by which the heat fluxes are defined as $\bar{S}_{\|}=\bar{S}_{\perp}=\gamma$. By means of these parameters the coefficients $a_{0,1,2}$ and $b_{0,1,2}$ are defined as

$$
\begin{aligned}
& a_{0}=1-j \eta^{2}, a_{1}=1-2 \gamma \eta-j \bar{\alpha} \eta^{2}, a_{2}=1+2 \gamma \eta-j \eta^{2} \\
& b_{0}=1-3 j \eta^{2}, b_{1}=2 \gamma \eta(\alpha-2)-2, b_{2}=3+4 \gamma \eta-3 j \eta^{2}
\end{aligned}
$$


With the above expressions and inserting Eqs. (9213) we obtain from Eqs. (10 12

$$
\begin{aligned}
& \frac{1}{\eta} \frac{\vec{v}}{c_{\|}}-\alpha \frac{\vec{k}}{k_{\|}}\left(\frac{a_{1}}{a_{0}} \frac{B^{\prime}}{B}+\frac{a_{2}}{a_{0}} \frac{(\vec{k} \cdot \vec{v})}{\omega}+\frac{\left(\vec{B} \cdot \vec{B}^{\prime}\right)}{4 \pi p_{\perp}}\right)+ \\
& +\frac{\vec{B}^{\prime}}{B}(\beta-\bar{\alpha})-\frac{\vec{B}}{B}\left[\left(\frac{b_{1}}{b_{0}}-\alpha \frac{a_{1}}{a_{0}}-2 \bar{\alpha}\right) \frac{B^{\prime}}{B}+\left(\frac{b_{2}}{b_{0}}-\alpha \frac{a_{2}}{a_{0}}\right) \frac{(\vec{k} \cdot \vec{v})}{\omega}\right]=0, \\
& \frac{\vec{B}^{\prime}}{B}-\frac{\vec{B}}{B} \frac{(\vec{k} \cdot \vec{v})}{\omega}+\frac{k_{\|}}{\omega} \vec{v}=0, \quad\left(\vec{k} \cdot \vec{B}^{\prime}\right)=0 .
\end{aligned}
$$

For the considered homogenous model we can place, without loss of generality, both vectors of the unperturbed magnetic field $\vec{B}$ and the wave vector $\vec{k}$ into the same plane, say $x-z$. Then the magnetic field perturbation vector $\vec{B}^{\prime}$ is in the perpendicular plane. Let the wave vector be along the $x$-axis. In this geometry we have $\vec{k}=(k, 0,0)$, $\vec{B}=\left(B_{x}, 0, B_{z}\right), \overrightarrow{B^{\prime}}=\left(0, B_{y}^{\prime}, B_{z}^{\prime}\right)$, and $\vec{v}=\left(v_{x}, v_{y}, v_{z}\right)$. Note that $B_{x}=B \cos \theta$ and $B_{z}=B \sin \theta$. Taking the $x, z$ and $y$ components of the vector Eqs. (19) we get

$$
\begin{aligned}
& q_{1} \frac{v_{x}}{c_{\|}}-q_{2} \tan \theta \frac{B_{z}^{\prime}}{B}=0, \frac{1}{\eta} \frac{v_{z}}{c_{\|}}-q_{3} \tan \theta \frac{v_{x}}{c_{\|}}+q_{4} \frac{B_{z}^{\prime}}{B}=0, \\
& \frac{B_{z}^{\prime}}{B}-\eta \tan \theta \frac{v_{x}}{c_{\|}}+\eta \frac{v_{z}}{c_{\|}}=0, \frac{1}{\eta} \frac{v_{y}}{c_{\|}}+(\beta-\bar{\alpha}) \frac{B_{y}^{\prime}}{B}=0, \frac{B_{y}^{\prime}}{B}+\eta \frac{v_{y}}{c_{\|}}=0 .
\end{aligned}
$$

Here $q_{4}=\beta-\bar{\alpha}-l_{2} q_{0}$,

$$
q_{1}=\frac{1}{\eta}-\eta \frac{\alpha}{l_{1}} \frac{a_{2}}{a_{0}}-q_{3}, \quad q_{3}=\eta\left(\frac{b_{2}}{b_{0}}-\alpha \frac{a_{2}}{a_{0}}\right), \quad q_{2}=\alpha \frac{a_{1}}{a_{0}}+\beta+l_{1} q_{0}, \quad q_{0}=\frac{b_{1}}{b_{0}}-\alpha \frac{a_{1}}{a_{0}}-2 \bar{\alpha} .
$$

The last two $y$-Eqs. (22) are separated from the others and give the dispersion relation of fire hose modes $\eta^{2}(\alpha+\beta-1)=1$ or

$$
\left(\frac{\omega}{k}\right)^{2}=v_{A}^{2}\left(1-\frac{p_{\|}-p_{\perp}}{2 p_{\mathrm{m}}}\right) \cos ^{2} \theta
$$

$p_{\mathrm{m}}$ is the magnetic pressure. The fire hose modes (further the label $f h$ is used for these modes) are prototypes of the Alfvén waves, and they become unstable if $\alpha+\beta<1$ or if $p_{\|}>p_{\perp}+2 p_{\mathrm{m}}$. The maximum of the instability growing rate corresponds to the parallel propagation case when $\cos ^{2} \theta=1$. These modes are incompressible, and they do not disturb the density of the plasma. The properties of these modes remain unchanged including the heat flux evaluation Eqs. (5-6).

The zero determinant of the first three $x-$ and $z$-equations of Eqs. (21) 22) gives the dispersion relation for the other modes:

$$
l_{2} q_{2}\left(\frac{1}{\eta}-q_{3}\right)-l_{1} q_{1}\left(\frac{1}{\eta^{2}}-q_{4}\right)=0
$$

\section{CGL mirror wave instability}

To obtain the wave modes based on the CGL-invariants we should set $\gamma=0$ and $j=0$ in the general dispersion Eq. (25). Then $a_{0}=a_{1}=a_{2}=b_{0}=1, b_{1}=-2, b_{2}=3$, and the dispersion equation is

$$
2\left(\frac{\omega}{k c_{\|}}\right)^{2}=\alpha+\beta+2 l_{1}+\alpha l_{2} \pm \sqrt{A}, \quad A=\left(\alpha+\beta+\alpha l_{2}-4 l_{1}\right)^{2}+4 l_{1} l_{2} \alpha^{2},
$$

which has been obtained by many authors [29,5]. Here the fast and slow mirror mode waves correspond to the plus and minus signs, respectively. Let the labels of these modes be $\mathrm{fm}$ and $\mathrm{sm}$. In many aspects the properties of these well-known CGL modes are similar to the usual MHD waves. For the parallel propagation case $\left(l_{1}=1, l_{2}=0\right)$ the squared phase velocities are $V_{f m}^{2}=V_{f h}^{2}=\alpha+\beta-1$ and $V_{s m}^{2}=3$ if $\alpha+\beta \geq 4$. In 
the opposite case $\alpha+\beta<4$ we have $V_{s m}^{2}=V_{f h}^{2}=\alpha+\beta-1$ and $V_{f m}^{2}=3$. In the perpendicular propagation case $\left(l_{1}=0, l_{2}=1\right) V_{s m}^{2}=V_{f h}^{2}=0$ and $V_{f m}^{2}=2 \alpha+\beta$. Note that here the phase velocities are normalized to $c_{\|}$.

However, there is a difference between the mirror modes and the MHD magnetosonic waves: the relation $V_{s m}^{2} \leq V_{f h}^{2} \leq V_{f m}^{2}$ between the phase velocities is not always valid. In some parameter ranges the slow modes may propagate faster than the Alfvénic fire hose modes [29,19]. This behavior is opposite to the isotropic MHD theory. The fast mirror modes are always stable $\left(V_{f m}^{2}>0\right)$ as $A>0$. However, in some parameter ranges the slow modes become unstable, $V_{s m}^{2}<0$. All of these properties can easily be obtained from Eq. (26). If both the fire hose and the mirror instabilities arise at the same time the growing rate of the first one is always greater, $\operatorname{Im}\left(V_{f h}\right) \geq \operatorname{Im}\left(V_{s m}\right)$. The slow mirror instability condition is

$$
l_{2} \alpha^{2}>3\left(\alpha+\beta+\alpha l_{2}-l_{1}\right) .
$$

In the parallel propagation case $\left(l_{1}=1, l_{2}=0\right)$ this passes to the fire hose instability condition $\alpha+\beta<1$ or $p_{\|}>p_{\perp}+2 p_{\mathrm{m}}$. For the quasi-perpendicular modes $\left(l_{1}=0, l_{2}=1\right)$ the mirror instability arises if $\alpha^{2}>3(2 \alpha+\beta)$ or $p_{\perp}^{2} / p_{\|}>6\left(p_{\perp}+p_{\mathrm{m}}\right)$. Compared to the kinetic theory the CGL theory gives the exact fire hose instability criterion, but the mirror instability conditions differs by a factor of 6 [55, 18, 35].

\section{Instabilities with heat fluxes}

Let us now study our general dispersion Eq. (25), obtained without using the CGL invariants. In this equation $j \equiv 1$ and generally $\gamma \neq 0$. This is a polynomial equation of 8 th order in the frequency of the fluctuations. For the parameter $\eta=c_{\|} k_{\|} / \omega$ the dispersion equation can be written in the form

$$
c_{8} \eta^{8}+c_{6} \eta^{6}+c_{4} \eta^{4}+c_{2} \eta^{2}+c_{0}+\gamma\left(c_{7} \eta^{7}+c_{5} \eta^{5}+c_{3} \eta^{3}\right)=0
$$

where for $l=l_{1}=\cos ^{2} \theta, s=\alpha^{2}(1-l)$,

$$
\begin{gathered}
r=l-\beta-\alpha(2-l), c_{8}=3(2 s+r), c_{7}=-4(3 s+r), c_{5}=4(s+r-l), \\
c_{6}=2 s\left(2 \gamma^{2}-5\right)+3(l-3 r), c_{4}=2 s+7 r-9 l, c_{3}=4 l, c_{2}=7 l-r, c_{0}=-l .
\end{gathered}
$$

Here all the coefficients are real, consequently, all solutions are real or conjugate complex. So, instead of the 4 th order biquadratic CGL dispersion equation we have deduced now the 8 th order Eq. (28) in the anisotropic MHD. With the initial heat fluxes, $\gamma \neq 0$, odd nonzero coefficients $c_{3}, c_{5}, c_{7}$ will result in wave propagation velocities depending on the propagation direction with respect to the magnetic field. We can expect prograde and retrograde wave modes. In the case of the CGL equations only two mirror modes can arise, the phase velocities of which are equal to each other in both directions with respect to the magnetic field. Let us first consider the most important limiting and special cases of Eq. (28) which can be solved analytically. It is useful to represent Eq. (28) as follows:

$$
\left(\eta^{2}-1\right)\left(\mu \eta^{2}-l\right)\left(3 \eta^{4}-6 \eta^{2}+1-4 \gamma \eta^{3}\right)-2 \alpha^{2}(1-l) \eta^{4} \wp=0
$$

where $\wp=3 \eta^{4}+\left(2 \gamma^{2}-5\right) \eta^{2}+1+2 \gamma \eta\left(1-3 \eta^{2}\right)$ and $\mu=\alpha(2-l)+\beta-l$.

\subsection{Parallel propagation}

In the case of wave propagation along the magnetic field $l=1, k_{\|}=k$, and the phase velocity normalized to the parallel sound speed $c_{\|}$is $V=1 / \eta$. In this case the dispersion Eq. 29] becomes

$$
\left(\eta^{2}-1\right)\left[\mu \eta^{2}-1\right]\left[3\left(\eta^{2}-1+\sqrt{2 / 3}\right)\left(\eta^{2}-1-\sqrt{2 / 3}\right)-4 \gamma \eta^{3}\right]=0,
$$

where $\mu=\alpha+\beta-1$. In contrast to the CGL case there appear two additional modes which are connected with the heat fluxes. The phase velocities of these two fast and slow thermal modes (the corresponding labels are $f t$ and $s t$ ) are between the modified fast and slow mirror modes (corresponding labels are $f m$ and $s m$ ). So in 
the phase diagrams these modes will be recognized by the following relation between the real parts of the phase velocities:

$$
V_{s m}^{2} \leq V_{s t}^{2} \leq V_{f t}^{2} \leq V_{f m}^{2}
$$

The place of fire hose mode velocity $V_{f h}^{2}$ in this relation is arbitrary depending on $\alpha, \beta$, and $l$. In Eq. 31) the relation ' $\leq$ ' means that a coincidence of the mode branches is possible in a resonance interaction domains, where the instability is developing.

In Eq. (30) the first two quadratic roots do not depend on the heat flux parameter $\gamma$. That means, these modes are symmetric with respect to the magnetic field direction. The last four solutions do depend on $\gamma$. That means, waves running along and against the magnetic field direction have different velocities. For an easy classification of the solutions let us consider the case $\gamma \rightarrow 0$. Here we should remember that $\gamma=0$ means only that the initial fluxes are zero, but the perturbed non-zero fluxes generated by the wave motions are described by Eqs. (14,15. Now we have four quadratic roots. It depends on the value of $\mu$ which root is related to which mode. Let $\mu<0.55$. Then the root $\eta^{2}=1-\sqrt{2 / 3}$ corresponds to the fast mirror mode with $V_{f m}^{2}=3(1+\sqrt{2 / 3}) \simeq 5.4$. The root $\eta^{2}=1$ corresponds to the fast thermal mode with $V_{f t}^{2}=1$. The other root $\eta^{2}=1+\sqrt{2 / 3}$ corresponds to the slow thermal mode with $V_{s t}^{2}=3(1-\sqrt{2 / 3}) \simeq 0.55$. The slowest mode is the slow mirror mode corresponding to the root $\mu \eta^{2}=1$, for which $V_{s m}^{2}=\alpha+\beta-1$. The fire hose modes coincide with the slow mirror mode, $V_{f h}^{2}=V_{s m}^{2}$. However, with increasing $\mu$ the fire hose mode can coincide with every of the obtained modes. For instance, if $\mu>5.4$ then $V_{f h}^{2}=V_{f m}^{2}$. Such a hierarchy between the phase velocities is similar to the CGL or MHD theories. In the considered case the instability is possible if $\mu<0$. This means only the slow mirror modes can be unstable. Other wave modes propagating along the magnetic field are stable.

However, we have ignored $\gamma$ in Eq. (30). With increasing $\gamma$ an additional instability is possible as well. This can easily be verified considering a large $\gamma \gg 1$. The 4 solutions are: $\eta_{1} \approx 4 \gamma / 3, \eta_{2} \approx(4 \gamma)^{-1 / 3}$, $\eta_{3,4} \approx(4 \gamma)^{-1 / 3} e^{ \pm 2 i \pi / 3}$. The phase velocities corresponding to these solutions are: $V_{1} \approx 3 / 4 \gamma \ll 1, V_{2} \approx$ $(4 \gamma)^{1 / 3} \gg 1, V_{3,4} \approx(4 \gamma)^{1 / 3}(-1 \pm i \sqrt{3}) / 2$. The two roots of $\eta^{2}=1$ give $V_{5,6}= \pm 1$. In the fire hose instability parameter range $(\mu<0)$ the roots of the last equation $\mu \eta^{2}=1$ correspond to $V_{7,8}= \pm i \sqrt{1-\alpha-\beta}$. Now we apply the relation Eq. (31) to the waves propagating along $(\operatorname{Re}(V)>0)$ and against $(\operatorname{Re}(V)<0)$ the magnetic field separately. For the positive phase velocities we have: $V_{s m}^{+}=\operatorname{Re}\left(V_{7}\right)=0, V_{s t}^{+}=V_{1} \ll 1, V_{f t}^{+}=V_{5}=1$, $V_{f m}^{+}=V_{2} \gg 1$. For the negative phase velocities we get: $V_{s m}^{-}=\operatorname{Re}\left(V_{8}\right)=0, V_{s t}^{-}=V_{6}=-1, V_{f t}^{-}=\operatorname{Re}\left(V_{3}\right)$, $V_{f m}^{-}=\operatorname{Re}\left(V_{4}\right)$. The squares of these negative phase velocities also satisfy Eq. (31). From here we conclude the role of the nonzero initial heat fluxes:

i) Retrograde thermal modes are faster than prograde thermal modes, $\left|V_{s t}^{-}\right| \gg V_{s t}^{+}$and $\left|V_{f t}^{-}\right| \gg V_{f t}^{+}$. For the fast mirror waves we have the opposite case: prograde modes are twice faster than the retrograde modes, $V_{f m}^{+}=2\left|V_{f m}^{-}\right|$. Slow mirror modes remain symmetric. If we let the parameter $\gamma$ go back to zero, all the discrepancies between the same modes disappear.

ii) Instabilities have a resonance origin. Instabilities grow when two (maybe even more) phase velocities coincide. The aperiodic fire hose instability develops when the velocities of the slow mirror modes are zero, $V_{s m}^{+}=V_{s m}^{-}=$ 0 . The periodic instability (non-zero real frequency) appears, when the retrograde fast thermal and mirror modes are in resonance, $V_{f t}^{-}=V_{f m}^{-} \neq 0$. Depending on the values of $\gamma$ the grow rate of the thermal mirror instability may be greater than the fire hose growing rate.

\subsection{Quasi-perpendicular propagation}

In the case of quasi-perpendicular propagation $(l \rightarrow 0)$ inserting $\eta=l^{1 / 2} X$ into Eq. (28) results in

$$
l^{3} c_{8} X^{8}+l^{2} c_{6} X^{6}+l c_{4} X^{4}+c_{2} X^{2}-1+l^{3 / 2} \gamma\left(l c_{7} X^{4}+c_{5} X^{2}+4\right) X^{3}=0,
$$

where the coefficients $c_{2-8}$ are the same as in Eq. (28) if we insert there $l=0$ and the phase velocity $V=1 / X$. The first two solutions of this equation for $l=0$ are $X^{2}=1 / c_{2}=1 /(2 \alpha+\beta)$. These are equal to the stable CGL fast mirror modes with the phase velocity $V_{1,2}= \pm \sqrt{2 \alpha+\beta}$. To find the other 6 solutions of Eq. (32) we introduce the new variable $X=Y / \sqrt{l}$. Then the equation for $Y$ is

$$
c_{8} Y^{6}+c_{6} Y^{4}+c_{4} Y^{2}+c_{2}+\gamma Y^{3}\left(c_{7} Y^{2}+c_{5}\right)=0 .
$$


Let us consider the case $\gamma \rightarrow 0$. For $Z=Y^{2}$ we have now

$$
c_{8} Z^{3}+c_{6} Z^{2}+c_{4} Z+c_{2}=0 .
$$

Remind that the remaining 6 phase velocities are expressed by three exact solutions of this cubic equation, $V_{3-8}=$ $\pm \sqrt{l / Z_{1,2,3}}$. The known analytical solutions of Eq. (34) are still cumbersome. But for some parameter limits we can give these solution in simple expressions. Let $\sigma=(2 \alpha+\beta) /\left(2 \alpha^{2}\right)$. Then Eq. 34 is

$$
3(1-\sigma) Z^{3}+(9 \sigma-5) Z^{2}+(1-7 \sigma) Z+\sigma=0 .
$$

We will consider three limit cases: $\sigma \approx 1, \sigma \ll 1$, and $\sigma \gg 1$.

i) Expansions of the solutions around $\sigma=1$ give $Z_{1,2} \approx(3 \pm \sqrt{5}) / 4$ and $Z_{3} \approx 4 / 3(\sigma-1)$. Correspondingly the phase velocities are $V_{3-6} \approx \pm \sqrt{l(3 \pm \sqrt{5})}$ and $V_{7-8} \approx \pm \sqrt{3 l(\sigma-1) / 4}$. The first 4 solutions are stable as $V_{3-6}^{2}>0$, but the last ones may become unstable $V_{7-8}^{2}<0$ if $\sigma<1$ is obeyed. This corresponds to the mirror instability criterion well known from kinetic plasma physics [57, 30, 18], $2 \alpha+\beta<2 \alpha^{2}$ or

$$
\frac{p_{\perp}^{2}}{p_{\|}}>p_{\perp}+p_{\mathrm{m}} .
$$

Contrary to the CGL case the factor 6 does not appear on the r.h.s. of this condition.

Expanding the dispersion equation around small $l \ll 1$ we obtain the growing rate of the mirror instability

$$
\frac{\omega}{k c_{\|}} \approx \pm i \frac{3 l}{2} \sqrt{1-\sigma-l \frac{(\alpha-1)(2 \alpha+1)}{2 \alpha^{2}}} .
$$

The maximum of this growing rate is $\sqrt{6} \alpha(1-\sigma) /[4 \sqrt{(\alpha-1)(2 \alpha+1)}]$ which corresponds to a critical angle of $l_{c}=\alpha^{2}(1-\sigma) /[(\alpha-1)(2 \alpha+1)] \leq 1$. Qualitatively this result for the fluid approximation is in good agreement with similar kinetic results, such as for the guiding center theory [32], the low frequency analytical limit of kinetic turbulence [57], and the more exact numerical results for different kinds of particle distribution functions [14].

ii) The limit case $\sigma \ll 1$ corresponds to $\alpha \gg 1$ or $p_{\perp} \gg p_{\|}$(hot particle across the magnetic field limit). In this case $V_{3-6} \approx \pm \sqrt{l(5 \pm \sqrt{13}) / 2}$ and $V_{7-8} \approx \pm i \sqrt{l / \sigma}$. Again the first 4 mode solutions are stable, the last two modes become unstable. The growing rate of these modes may be smaller or larger depending on the ratio $l / \sigma$.

iii) The limit $\sigma \gg 1$ is also interesting. This limit corresponds to $\alpha \ll 1\left(p_{\perp} \ll p_{\|}-\right.$hot particles along the magnetic field) or $\beta \gg 1-$ strong magnetic field. In this case we have only stable modes: $V_{3-6} \approx \pm \sqrt{l(3 \pm \sqrt{6})}$ and $V_{7-8} \approx \pm \sqrt{l}$.

If we apply Eq. (31) to the deduced phase velocities we obtain for $\sigma \approx 1$ that $V_{f m}^{2}=V_{1,2}^{2}, V_{f t, s t}^{2}=V_{3-6}^{2}$, and $V_{s m}^{2}=V_{7,8}^{2}$. These definitions are the same as those for the case $\sigma \gg 1$, but for $\sigma \ll 1$ this definition is strongly depending on the ratio of the two small parameters $l / \sigma$.

For large $\gamma \gg 1$ it can easily be shown from the asymptotical solutions of Eq. (33) that the mirror instability condition (36) remains unchanged.

\subsection{Oblique propagation}

For the oblique propagation case $(l \neq 1$ and $l \neq 0)$ it becomes more difficult to analyze Eq. 28). However, there are some important limit cases for which simple analytical solutions are possible.

\subsubsection{The case $\alpha \ll 1$}

This case is similar to the parallel propagation case. Here Eq. 29) is reduced to Eq. (30) with the difference that the parameter $\eta$ contains $l$ and the phase velocity $V^{2}=l / \eta^{2}$. Besides, instead of the equation $\mu \eta^{2}=1$ we have $\mu \eta^{2}=l$ with $\mu=\beta-l$. That means slow mirror waves become unstable at oblique propagation: $V_{7,8}= \pm \sqrt{\beta-l}$. Instability appears if $\beta<l$. 


\subsubsection{The case $\alpha \gg 1$}

In this case $V=\sqrt{l} / \eta$ and the 4 solutions of Eq. (28) are

$$
V^{2} \approx \frac{\alpha}{2}\left[1+l_{2} \pm \sqrt{\left(1+l_{2}\right)^{2}+8 l l_{2}}\right]
$$

where $l_{2}=1-l$. The ' + ' and '-' solutions are fast and slow mirror modes, respectively. These modes do not depend on $\gamma$, and they are symmetric with respect to the propagation direction. The fast modes are stable, but unstable are the slow modes with large growing rate. The instability develops aperiodically.

The other 4 solutions for the thermal modes are described by the equation

$$
3 \eta^{4}+\left(2 \gamma^{2}-5\right) \eta^{2}+1+2 \gamma \eta\left(1-3 \eta^{2}\right)=0
$$

For $\gamma \ll 1$ the thermal modes are stable, $V^{2} \approx l(5 \pm \sqrt{13}) / 2>0$. With increasing $\gamma$ instability appears. In the limit $\gamma \gg 1$ the prograde modes remain stable with $V \approx \sqrt{l}(3 \pm \sqrt{3}) / 2 \gamma$, but the retrograde modes become strongly unstable with

$$
V \approx \gamma \sqrt{l}(-1 \pm i)
$$

The real parts of the phase velocities of the fast and slow thermal modes coincide, and a periodical thermal instability develops.

\subsubsection{The case $\beta \gg 1$}

A strong magnetic field $\beta=2 p_{m} / p_{\|} \gg 1$ (reverse plasma beta) is an often used special case. In this case the solutions of Eq. (28) are also simple. The fast and slow mirror modes are symmetrical and stable, $V_{f m}^{2} \approx \beta$ and $V_{s m}^{2} \approx l$. If the heat flux parameter $\gamma \ll 1$ then both the fast and the slow thermal modes are symmetric and stable too, $V^{2} \approx 3 \pm \sqrt{6}$. However, with increasing $\gamma$ there appears an asymmetry and an instability of the thermal modes. For $\gamma \gg 1$ the prograde modes are asymmetric but stable, $V_{f t} \approx \sqrt{l}(4 \gamma)^{1 / 3}$ and $V_{s t} \approx 3 \sqrt{l} /(4 \gamma)$. The phase velocities of the two retrograde modes become equal and they are unstable: $V \approx \sqrt{l}(4 \gamma)^{1 / 3}(-1 \pm i \sqrt{3}) / 2$.

\subsubsection{The case $\gamma \gg 1$}

This special case of a large heat flux parameter, but moderate parameters for the rest is also of some theoretical interest. Assuming $l \neq 0$ and $l \neq 1$ we obtain that the two fast mirror modes are asymmetrical and stable: $V_{f m}^{ \pm} \approx \pm l^{1 / 2}[2 \gamma(g \pm l) / l]^{1 / 3}$, where $g=\left(l^{2}+\alpha^{2} l l_{2}\right)^{1 / 2}$, and the signs ' \pm ' correspond to the propagation direction on magnetic field. For the two slow mirror modes we have

$$
V_{s m}^{ \pm} \approx \frac{\sqrt{l}}{2 \gamma \nu_{1}}\left(3 \nu_{1}-\nu_{2} \pm \sqrt{3 \nu_{1}^{2}+\nu_{2}^{2}-3 \nu_{1} \nu_{2}}\right) .
$$

Here $\nu_{1}=\alpha^{2}(1-l), \nu_{2}=\alpha(2-l)+\beta-l$. As the expression under the square root is positive these modes are stable. The 4 thermal modes become unstable. For the prograde two thermal modes

$$
V_{f t, s t}^{+} \approx l^{1 / 2}[2 \gamma(g-l) / l]^{1 / 3}(1 \pm i \sqrt{3}) / 2
$$

and for the two retrograde thermal modes we have

$$
V_{f t, s t}^{-} \approx l^{1 / 2}[2 \gamma(g+l) / l]^{1 / 3}(-1 \pm i \sqrt{3}) / 2 .
$$



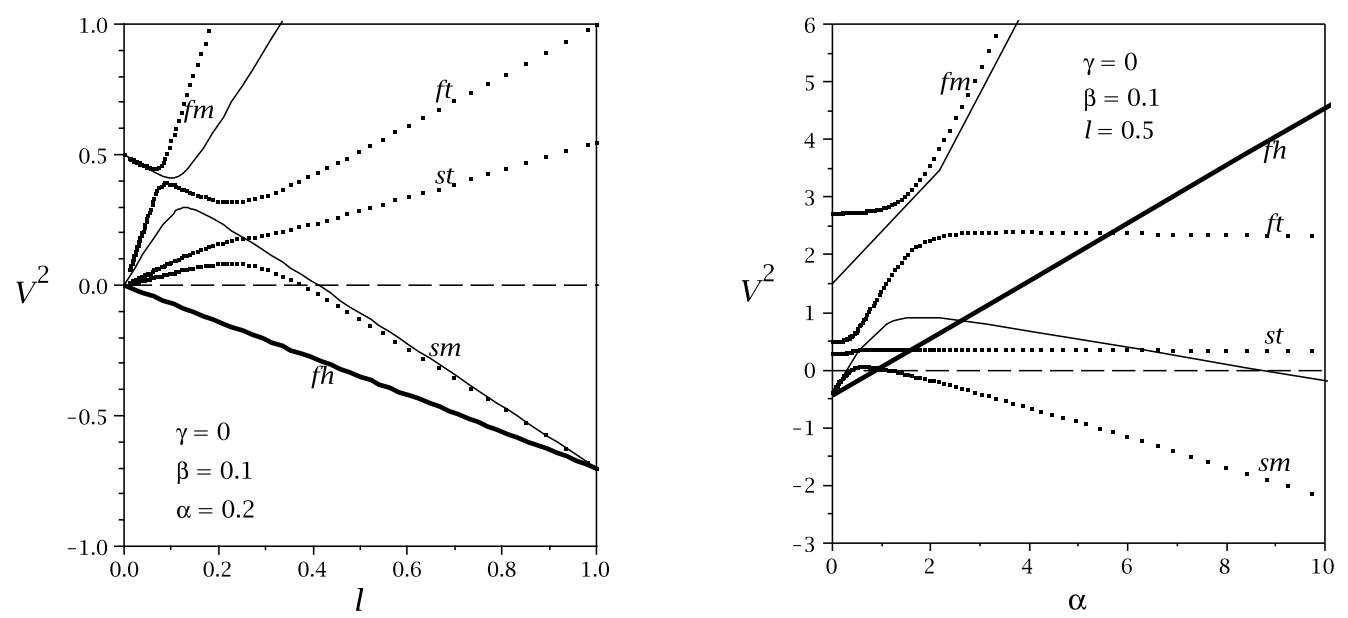

Fig. 1 The normalized phase velocity squared as function of the wave propagation angle $l=\cos ^{2} \theta$ (left-hand picture) and of the anisotropy parameter $\alpha=p_{\perp} / p_{\|}$(right-hand picture) for absent initial heat fluxes, $\gamma=0$. In the area below the dashed line where $V^{2}<0$ the modes become unstable for $\operatorname{Re}(\omega)=0$. The dotted lines are the 4 wave mode solutions of the dispersion Eq. (28). The 2 thin solid curves are the CGL fast and slow mirror modes. The thick solid line is the classic fire hose mode. The labels at the curves correspond to the mode classification: $f m$ - fast mirror, $s m$ - slow mirror, $f t$ - fast thermal, st - slow thermal, and $f h$ - fire hose modes.
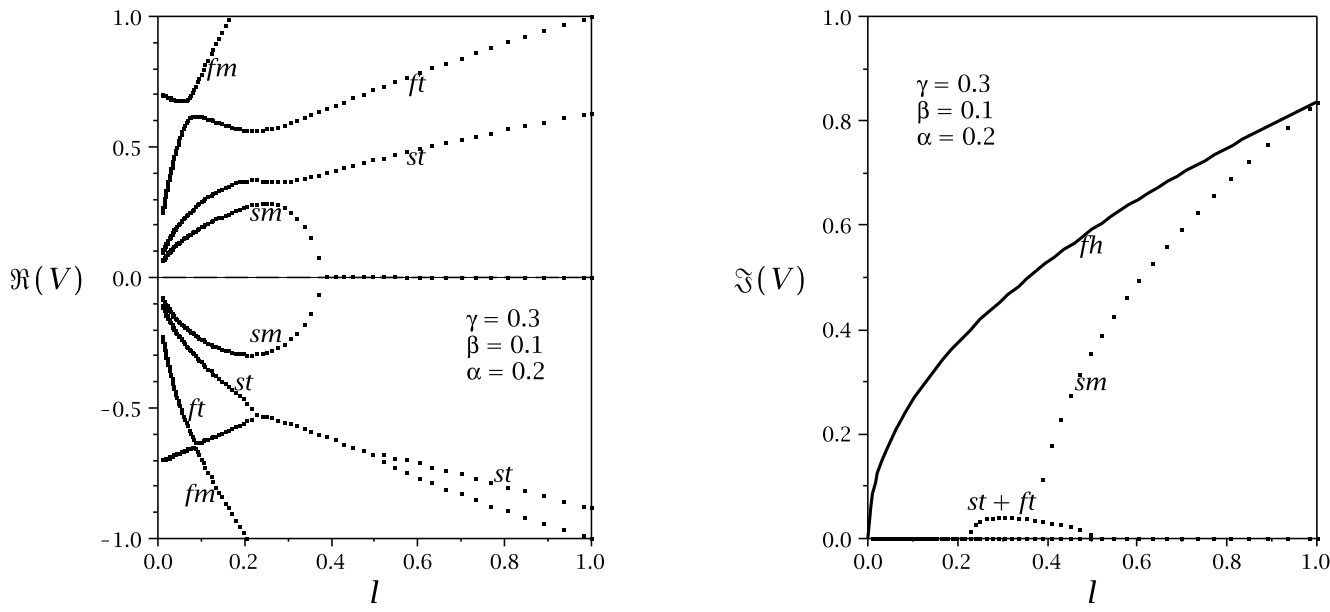

Fig. 2 The same as the left-hand picture of Fig. 11 but with non-zero heat fluxes, $\gamma=0.3$. The left-hand picture is for the phase velocities $\operatorname{Re}(\omega) / k c_{\|}$, the right-hand picture for the growing instability rates $\operatorname{Im}(\omega) / k c_{\|}$in dependence on the propagation angle parameter $l$. Instability arises when the phase velocities of the different modes in the left-hand picture coincide. In the right-hand picture the shown fire hose and slow mode instabilities are aperiodical $(\operatorname{Re}(\omega)=0)$, while the thermal instability is periodical $(\operatorname{Re}(\omega) \neq 0)$.

\subsubsection{Some numerical examples}

The coefficients of the dispersion Eq. (28) depend on four parameters. In realistic space plasmas the values of these parameters cover wide ranges: $\alpha>0, \beta>0, \gamma>0$, and $0 \leq l \leq 1$. Here we cannot illustrate all interesting parameter ranges, but we will show some typical examples for high plasma-beta $(=2 / \beta)$, which cannot be considered asymptotically. The first two pictures shown in Fig. 1 1 are normalized squared phase velocities $V^{2}=$ $\omega^{2} / k^{2} c_{\|}^{2}$ in the symmetrical case of $\gamma=0$. In these pictures our results (the 4 dotted curves) are compared with the well known CGL modes (the 2 thin curves). It is seen that both CGL modes are strongly modified, especially the slow mirror mode. The left-hand picture demonstrates the fire hose instability development when $\alpha+\beta<1$. 

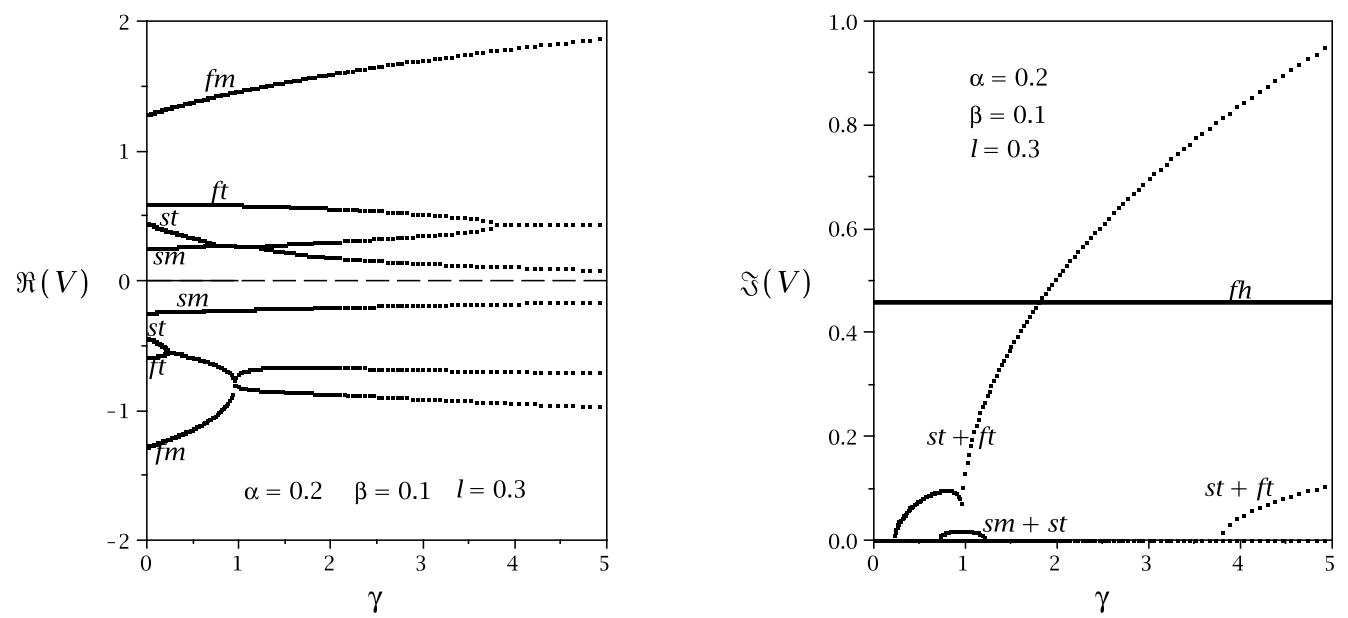

Fig. 3 Phase velocities (left-hand picture) and growing instability rates (right-hand picture) as functions of the heat flux parameter $\gamma$. Prograde modes $(\operatorname{Re}(V)>0)$ become unstable in two ranges of $\gamma: \gamma \approx 1$ where slow mirror and slow thermal modes are in resonance (the label $s m+s t$ in the right-hand picture) and $\gamma>3.8$ where two thermal modes are in resonance (the label $s t+f t$ in the lower right corner of the right-hand picture). The larger instability rate with label $s t+f t$ is due to the resonance of the two retrograde thermal modes. The fire hose mode $(f h)$ does not depend on $\gamma$.

With a decrease of the propagation angle both unstable slow modes tend to the fire hose mode and they are equal in the parallel propagation. For all angles the fire hose instability is dominant compared to the slow mode mirror instability. The right-hand picture shows the fire hose instability disappearing with increasing $\alpha$. Here we see the strong differences between the slow modes in the CGL and the new theory. The modified slow modes are highly unstable for $\alpha>1$. If the initial heat fluxes are absent $(\gamma=0)$ both additional thermal modes are stable.

The second set of two pictures shown in Fig. 2 are similar to those on the left-hand side of Fig. 1 (growing of fire hose instability), but now with non-zero initial heat fluxes, $\gamma \neq 0$. The slight deviation of $\gamma$ from zero has a strong influence on the retrograde thermal modes. In some range of the parameter $l$ two fast and slow thermal modes resonantly interact. In this region the phase velocities are equal and a periodical thermal instability develops. For the considered parameter values and small $\gamma$ the other modes are only slightly changed. The aperiodical fire hose and the slow mode mirror instabilities also arise when the prograde and retrograde phase velocities become equal to zero. The found new thermal instability arises strongly for oblique propagation. For the considered parameters this range corresponds to $0.2<l<0.5$, where the instability rate has a maximum. As seen from the right-hand picture the fire hose instability is still dominant.

In the last two pictures in Fig. 3 we show how the new thermal instability is changed with increasing $\gamma$. We choose $l=0.3$ where in Fig. 2 the thermal instability has its maximum. With $\gamma$ the growing rate of these retrograde thermal modes increases very sharply, and it becomes larger than the growing rate of the fire hose instability. To compare it with the fire hose instability in the right-hand picture the level of the growing rate of the fire hose instability is shown too. Here appear also the instabilities of the prograde modes, but with smaller growing rates. Slow thermal modes interact at first with the slow mirror modes close to $\gamma \approx 1$, then for higher $\gamma>4$ they are in resonance with the fast thermal modes.

\section{Compressible fire hose instability}

The classical incompressible fire hose instability arises if $\eta^{2}=-a$ and $a=1 /(1-\alpha-\beta)>1$ are obeyed, see Eq. (24). Near the threshold of this instability $a \gg 1$. This is an aperiodical instability, that means $\operatorname{Re}(\omega)=0$. Here we show that the dispersion equation for the compressible modes Eq. 28) can reach a similar solution at the threshold of the fire hose instability but with a small non-zero real frequency. For this aim we will search the solution of Eq. 28, in the form

$$
\eta^{2}=-a(1+i \epsilon)
$$




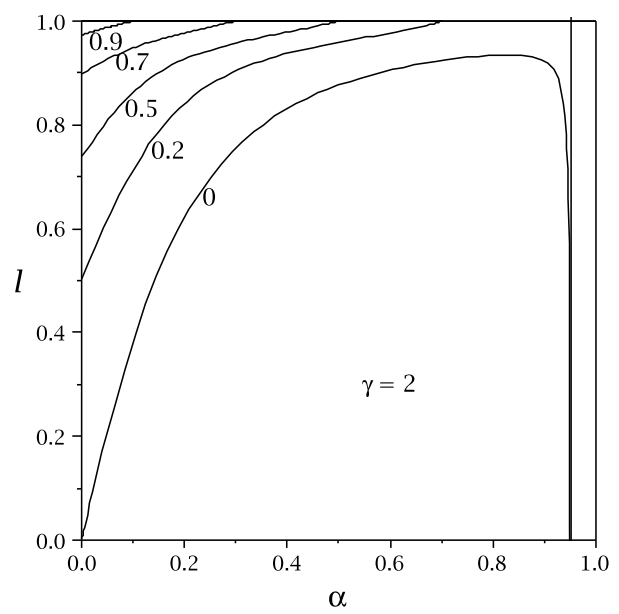

Fig. 4 Compressible fire hose instability domains in dependence on $\alpha, l$, and $\beta$ (the numbers at the curves) for given $\gamma=2$.

where $\epsilon$ is a new unknown which is real. For simplicity let $|\epsilon| \ll 1$ and $\eta \approx \mp i a^{1 / 2}(1+i \epsilon / 2)$. Then Eq. (28) is split into two equations which correspond to its real and imaginary parts. The first one defines the parameter $\epsilon$ :

$$
\epsilon \approx \pm \gamma a^{3 / 2} \frac{c_{7} a^{2}-c_{5} a+c_{3}}{4 c_{8} a^{4}-3 c_{6} a^{3}+2 c_{4} a^{2}-c_{2} a}
$$

Near the instability threshold where $a \gg 1$ we get a simple relation for $\epsilon$ :

$$
\epsilon \approx \mp \frac{\gamma}{3 \sqrt{a}} \frac{1+\alpha-3 \alpha^{2}}{(1-\alpha)(2 \alpha+1)} .
$$

Here we should remember that $\alpha<1$. The second equation is

$$
c_{8} a^{4}-c_{6} a^{3}+c_{4} a^{2}-c_{2} a+c_{0}=\frac{\gamma^{2} a^{3}}{2} \frac{\left(c_{7} a^{2}-c_{5} a+c_{3}\right)\left(7 c_{7} a^{2}-5 c_{5} a+3 c_{3}\right)}{4 c_{8} a^{4}-3 c_{6} a^{3}+2 c_{4} a^{2}-c_{2} a} .
$$

This is a quadratic equation for $l=\cos ^{2} \theta: d_{1} l^{2}+d_{2} l+d_{3}=0$. The coefficients $d_{1,2,3}$ are real functions of the parameters $\alpha, \beta$, and $\gamma$. The cumbersome expressions of these coefficients can be obtained easily from Eq. 47). In Fig. 4 we show these solutions in the range $0 \leq l \leq 1$ for $\gamma=2$. Only in the fire hose instability parameter values, when $\alpha<1$ and $\beta<1$, we get $l$ in the range $0 \leq l \leq 1$. The $l(\alpha, \beta)$ picture is not strongly changed for values $0 \leq \gamma \leq 2$. The quasi-parallel propagation of $l \approx 1$ is easily described by Eq. (29). For the found $l(\alpha, \beta, \gamma)$ the compressible periodical fire hose instability can develop in this way as described by Eq. (44) with $\epsilon(l)$ defined by Eq. (45). $\epsilon$ strongly depends on $\gamma$. For absent heat fluxes, $\gamma=0$, we have $\epsilon=0$ and the found oblique instability disappears. The complex phase velocity in this instability is defined as

$$
V \approx \pm(\epsilon / 2+i) \sqrt{l / a}
$$

Formally this formula is the same as that for the incompressible fire hose modes if we set $\gamma=0$. The difference between both modes is that the first one can develop for any $l$ in the given $\alpha$ and $\beta$ ranges, but the second fire hose instability develops only for one angle of propagation defined by $l(\alpha, \beta, \gamma)$. Taking into account Eq. (48) that $\operatorname{Re}(V)<0$. So the second fire hose instability is the result of the resonantly coupling of the retrograde thermal modes.

We think that the found second instability is analogous to the earlier found kinetic oblique fire hose instability [21]. Both the incompressible and the compressible instability growing rates become comparable to each other near the threshold of the fire hose instability. At the given high proton plasma beta $(=2.8)$ the oblique compressible fire hose instability rate at the angle $\theta=53^{\circ}$ reaches (or even slightly exceeds) the maximum of the incompressible fire hose instability rate in parallel propagation. We got here a possibility to test this proton 

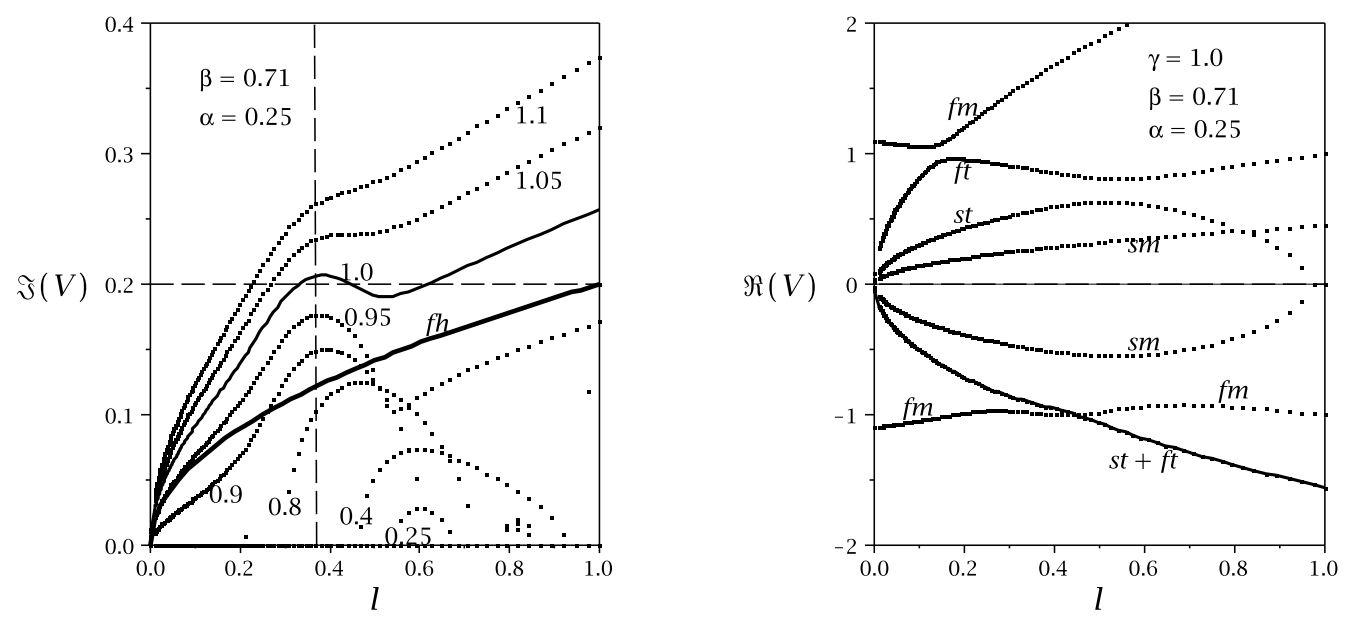

Fig. 5 Left-hand picture: compressible fire hose instability growing rate as function of $l$ and $\gamma$ (numbers at the curves). The thick solid curve is the growing rate of the incompressible fire hose instability, and the horizontal dashed line is the level of its maximum for parallel propagation $(l=1)$. The vertical dashed line corresponds to the angle $\theta=53^{\circ}(l=0.36)$ when the growing rates of the two kinetic fire hose instabilities become comparable to each other. For fluid instabilities this condition is obeyed for $\gamma \approx 1$. Right-hand picture: phase velocities for the case of $\gamma=1$.

anisotropy instabilities in our fluid approximation. We take the same parameters. In our definitions $\beta=0.71$, $l=0.362$, and $\alpha=0.25$ (close to the threshold). However, we have one free parameter - the parameter of the heat fluxes $\gamma$. Of course, in the kinetic consideration such a parameter does not exist. So this free parameter has to be varied to fit the results of the kinetic study. For this calculations the condition $\epsilon \ll 1$ is not used in Eq. (44). The results are shown in Fig. 5. It is seen from the left-hand picture that an analogous situation is reached for the fluid fire hose instabilities in the range $\gamma \lesssim 1$. The right-hand picture shows the phase velocities of the 8 modes for $\gamma=1$. The retrograde fast thermal modes are coupled at low $l$ with the slow thermal modes, and at higher values of $l$ these modes are in resonance with the fast mirror modes. For the value $l \sim 0.4$ of interest for us all 3 retrograde modes are in resonance. So the interaction of the 3 retrograde modes results in the appearance of the second kind of the fire hose instability.

\section{Mass density fluctuations}

The wave modes discussed above are compressible, they produce density perturbations. Here we shall write down further formulae for these perturbations which can be used in practice for identifying the wave motions.

$$
\begin{aligned}
& \alpha l_{2} \frac{a_{2}}{a_{0}} \frac{\rho^{\prime}}{\rho_{0}}=\left[l_{1}\left(\frac{1}{\eta^{2}}-\alpha-\beta+1\right)-l_{2}\left(\beta+\alpha \frac{a_{1}}{a_{0}}\right)\right] \frac{B^{\prime}}{B_{0}}, \\
& \frac{v_{\|}}{c_{\|}}=\frac{1}{\eta}\left(1-l_{2} \frac{1-\eta q_{3}}{1-\eta^{2} q_{4}}\right) \frac{\rho^{\prime}}{\rho_{0}}, \frac{v_{\perp}}{c_{\|}}=\frac{\sqrt{l_{1} l_{2}}}{\eta} \frac{1-\eta q_{3}}{1-\eta^{2} q_{4}} \frac{\rho^{\prime}}{\rho_{0}} .
\end{aligned}
$$

$v_{\|}$and $v_{\perp}$ are fluid velocity components along and across the magnetic field, $l_{1}=\cos ^{2} \theta, l_{2}=\sin ^{2} \theta$, and other parameters are defined by Eqs. (18) and (23). The inverse parallel component of the complex phase velocity $\eta=k_{\|} c_{\|} / \omega$ is defined as a solution of the dispersion relation Eq. (28). These formulae allow us to restore the full components of the fluid velocity and the magnetic field perturbation amplitudes if any components of velocity and density perturbations are known from observations.

Fig. 6 shows an example of the ratios of the parallel and perpendicular velocity amplitudes to the density perturbation amplitude. This case is equal to that on the right-hand picture of Fig. 5 where $\alpha=0.25, \beta=0.71$, and $\gamma=1$ is considered. Such a pictures gives direct information on the polarization of every type of wave modes. For instance, it is seen that all wave modes are almost longitudinally polarized at parallel propagation, $l=1$. It follows from the first relation of Eq. 49) (the picture is not shown here) that the usual isotropic MHD 

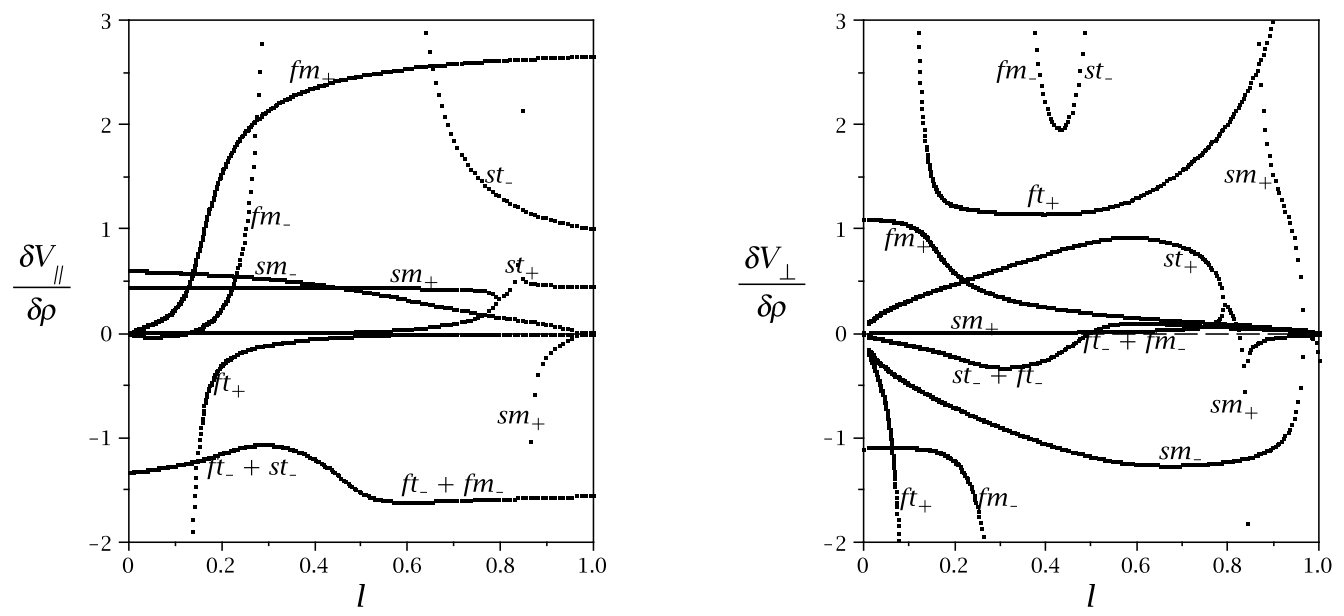

Fig. 6 Examples of fluid velocity and density perturbation amplitude relations in dependence on $l=\cos ^{2} \theta$ for given other parameters $\alpha=0.25, \beta=0.71$, and $\gamma=1$. Here $\delta \rho=\operatorname{Re}\left(\rho^{\prime} / \rho_{0}\right), \delta V_{\|}=\operatorname{Re}\left(v_{\|} / c_{\|}\right)$and $\delta V_{\perp}=\operatorname{Re}\left(v_{\perp} / c_{\|}\right)$. The last quantities are normalized fluid velocity components along and across the magnetic field. The labels at the curves correspond to the wave classification marks, and plus and minus at the indices of the labels correspond to prograde and retrograde wave modes, respectively.

relationship of $\delta \rho \delta B<0$ for the slow MHD mode and $\delta \rho \delta B>0$ for the fast MHD mode is not valid here. The sign of $\delta \rho \delta B$ can change in dependence on the parameters values.

\section{Discussion and Conclusions}

\subsection{Model equations}

Compared with a full kinetic model the fluid description of a plasma has the mathematical advantage of a smaller number of dimensions. Moreover, many observed dynamic phenomena in space plasmas are large-scale structures - already averaged over both temporal and spatial scales. This suggests to find ways for a description of a plasma as a fluid. It is easily possible in the case of a collision-dominated plasma which is described by a Maxwellian distribution function. In this case the usual isotropic MHD equations are received. The situation becomes much more complicated for a smaller frequency of the collisions between the particles of a hot magnetized plasma (such as space plasmas in most cases). Due to the magnetic field the collisionless plasma becomes anisotropic with respect of its local direction. To describe in this case the plasma as a fluid the transport model equations are deduced from the kinetic equations for the moments of the distribution function. These moments are such quantities as plasma mass density, fluid speed of the plasma, anisotropic thermal pressure, anisotropic thermal flux, etc. Basically, the number of these moments is infinite, and the equations of these moments are coupled among each other. However, if the conditions $\omega / \Omega_{B} \ll 1$ and $k r_{B} \ll 1$ are satisfied ( $\omega-$ frequency of perturbations, $\Omega_{B}$ - gyration frequency, $k$ - wave number, $r_{B}$-gyration radius), it is possible to break off the chain of these equations, if some additional conditions related to the given exact analytical form of the particle distribution function are fulfilled. This method is called the standard method of MHD ordering of the kinetic equations, and the resulting new equations are called "transport equations". However, the application of this ordering method has some subtleties - it is not trivial [50]. Depending on these and on additionally chosen conditions, the obtained transport equations can be different. By including higher order moments it is possible to increase the accuracy of the transport equations. With increasing order of the ordering the accuracy of the model equations also increases, but they become more complicated for the analysis. Even though these equations can never be complete without supposing additional conditions, they describe well such phenomena as Alfvénic and acoustic (electronic and ionic) waves, they can include such an important kinetic effect as Landau damping. However, depending on the order of ordering such kinetic effects as drift-waves and other micro-instabilities can be lost. Besides, to deduce the transport equations an exact analytical type of the function of particle distribution 
with anisotropic temperatures, e.g. bi-Maxwellian or $k$-distributions, is required. That means, the fine structure of the realistic distribution functions and the related microphysics are ignored.

Among these model equations the CGL equation are most simple with respect to the included moments and the order of ordering. The heat flux tensor in these equations is ignored at all. Strictly speaking, i.e. the phase speed of the perturbations should be much larger than the thermal velocity of the particles. Such condition are met for Alfvénic modes, but for acoustic modes this condition is impracticable. The inclusion of a heat flux tensor to the equations was carried by many authors [453 39]. Results of our studies in the present paper (types of instabilities, conditions of their existence, thresholds and values of growing rates of instabilities, comparisons of these with the corresponding results for mirror and ion-acoustic instabilities in the low-frequency kinetic approach) let us come to the conclusion that the used equations derived by Ramos [50] are more correct.

To sum it up it can be said that we have used more complete fluid transport equations describing the macroscopic behavior of a magnetized anisotropic collisionless plasma. In particular, we include heat fluxes and their evolution which, contrary to the CGL-MHD model, are a basic feature and cannot be ignored. We consider only two parallel heat fluxes corresponding to parallel and perpendicular thermal motions of particles. Perpendicular heat fluxes have been neglected.

\subsection{Mirror instability}

In strongly magnetized and weakly collisional turbulent plasmas the anisotropy of the pressure develops in a spontaneous way [51,52]. In a high-beta plasma that triggers a number of instabilities, above all firehose and mirror [8, 48, 57, 6, 17]. The nonlinear evolution of the instabilities should have the tendency to compensate on the average the pressure anisotropies generated by the turbulence. Thus the development of the instability of the modes further changes the distribution function, tending to make it more isotropic, or to strengthen the magnetic structurization. If $p_{\perp}>p_{\|}$, there appear two kinds of electromagnetic instability: ion-cyclotron instability at frequencies $\omega<\Omega_{B i}\left(\Omega_{B i}-\right.$ ion cyclotron frequency) and magneto-mirror instability at a very low frequencies, $\omega \approx 0$. Because there exist numerous observations of low-frequency turbulence in magneto-active plasmas, for instance in magnetosheaths, in solar wind, and in cometary comas (see references in [15]) the mirror instability was studied theoretically in detail. It was shown that in ionic high-beta plasma the mirror modes become unstable if an anisotropy indicator $p_{\perp} / p_{\|}-1$ exceeds some critical value [8, 57]. This instability causing a local deformation of the magnetic field makes the plasma spatially inhomogeneous. It occurs because a part of the particles captured in "weak mirror traps" subdivides the distribution of particles into passing and trapped species [31].

The fluid analogy of the kinetic mirror instability has a similar simple description [17, 56]. Basically, the instability occurs because at low frequencies the changes of the perpendicular pressure of the plasma and of the magnetic field occur in opposite phases. Really, as follows from Eq. (13), for $\omega \rightarrow 0$ neglecting small density perturbations, $p_{\perp}^{\prime} \sim-p_{\perp}\left(\frac{p_{\perp}}{p_{\|}}-1\right) \frac{B^{\prime}}{B}$. Hence, in those places of the plasma where $p_{\perp}>p_{\|}$a decrease of the plasma pressure increases locally the intensity and the pressure of the magnetic field. Meeting the condition $\frac{p_{\perp}}{p_{\|}}-1>\frac{p_{\mathrm{m}}}{p_{\perp}}$ the force (caused by the total pressure) in perpendicular direction decreases. Thus, the increase in intensity of the magnetic field locally reduces the total pressure which, in its turn, pushes together the magnetic field lines even more, i.e. leads to a further growth of the magnetic field. It causes instability. From the invariance of the first magnetic moment of the plasma follows that the energy of the particles will simultaneously grow in the direction perpendicular to the magnetic field. From the conservation of total energy follows that the parallel energy should decrease accordingly. Under these conditions (when the magnetic moment and the total energy are conserved) the plasma will naturally flow from an area with high magnetic field intensity to an area with a weak magnetic field. That means there is a conversion of perpendicular to parallel energy. It looks like an acceleration of particles along the magnetic field caused by a certain force. The name of this force is magneto-mirror. But this force is a pseudo-force as there is only a swapping of energy from a perpendicular into a longitudinal direction, the total energy doesn't change. If the instability criterion is not fulfilled the fluid mirror modes show an oscillatory behavior.

Contrary to the fluid description of the mirror instability in the kinetic description not all particles equally react to the magnetic field changes [55]. Particles with small parallel speed "do not feel" the mirror force to the same degree as the particles with larger parallel speed. In this way with changing magnetic field its energy is not conserved, and the perpendicular pressure changes synchronously with the magnetic field change. Unlike the 
fluid approach in the kinetic treatment the mirror modes become non-oscillating (exponentially damped) if the instability criterion is not satisfied. This is because of the resonant origin of the kinetic mirror instability [54].

We can compare the growing rate of the mirror instability in our fluid description Eq. 37] with the different kinetic estimates. There is good agreement. Note that the more exact numerical calculations of the kinetic growing rate for bi-Maxwellian plasma [12] show that a maximum of the growth rate occurs at $k_{\perp} r_{B i} \sim 1$, well above the threshold, and $\Gamma_{\max } \sim k_{\|} v_{T i \|}$.

We focused our interest on the properties of the mirror modes because of the high probability of their realization in practice. The properties of the other modes, fire hose and thermal modes, are well known. The first one is the prototype of Alfvénic waves and the second one is analogous to the ionic magnetic sound in the kinetic approach. At places of a crossing of wave branches we have a mixing of modes, i.e. there is a resonant interaction of wave modes. As usually in hydrodynamics if we consider the anisotropy of the plasma as well as its spatial inhomogeneity, these points of mode intersection will introduce singularities into the wave equations. Unlike the kinetic approach where wave modes can grow or fade as a result of a resonance of particles and fluctuations, in the fluid description it occurs as the result of resonant interaction of different modes.

In the present paper the linear instability problem is investigated in a homogenous, unlimited plasma. The classic incompressible fire hose modes are not influenced by the heat fluxes, and their instability criterion is the same as that in kinetic theory. However, the two CGL mirror modes are strongly modified by the heat fluxes, and there appear two additional thermal branches. These thermal modes result from including the two dynamic evolution equations of the thermal fluxes. In the initial state nonzero heat fluxes are supposed, $\gamma \neq 0$. However, even for zero initial heat fluxes $(\gamma=0)$ the thermal modes appear. The deduced 8-th order polynomial dispersion equation describes the interaction of all of the 4 types of compressible modes and their stability. If the initial heat fluxes along the magnetic field $(\gamma \neq 0)$ are included, the mode dispersion behavior and the instability criteria are different for the same type of modes running along and backward with respect to the magnetic field.

To sum it up it can be said that the main shortages of the CGL fluid theory have been removed. It is shown that the fluid slow mirror instability criterion is the same as that in the kinetic theory: $p_{\perp}^{2} / p_{\|}=p_{\perp}+p_{\mathrm{m}}$. We have shown that in some selected ranges of the parameters $\alpha$ (parameter of pressure anisotropy), $\beta$ (parameter of magnetic field), and $\gamma$ (parameter of initial heat flux) in the plasma there exists such a propagation angle $(l=$ $\cos ^{2} \theta$ ) in which at the same time two kinds of fire hose instability can develop. The discovered new instability is compressible, slightly periodical $(\operatorname{Re}(\omega) \neq 0)$, and it has a larger growing rate than the incompressible fire hose instability for parallel propagation. It seems that these modes are analogous to the two kinds of fire hose instabilities found in kinetic theory [24 47/37/21]. This new instability develops when the three retrograde modes (two thermal and fast mirror) interact resonantly.

We found a strong dependence of the growth rate on the parameters $\alpha, \beta, \gamma$ and $l$. There appear different unstable and stable wave branches simultaneously within the given parameter ranges. Only the mode with the highest growth rate will dominate, and after some exponential growth the nonlinear stage of the instability should be considered. It is of basic importance for the found instabilities that in the collisionless plasma there is a plasma pressure anisotropy that is kinetically supported. The origin of this pressure imbalance is not important for our fluid approximation; it is the background of large-scale flows only. In principle many kinds of kinetic wave turbulence can support such a pressure anisotropy, the existence of which in the considered plasma situation is shown by observations.

\subsection{Solar wind}

We think that the discovered wave modes and their instabilities in the anisotropic fluid approximation are interesting for those plasmas for which the approximation for a magnetized hot plasma with rare collisions can be applied. Important candidates for such conditions are the solar wind and the solar corona plasma. Macroscopic turbulence observed in the solar wind [41] and in the stable coronal turbulent background (appearing in the nonthermal broadening of coronal emission line profiles [3]) may be a consequence of these instabilities. Moreover, it is now generally accepted that the observed large ion temperature anisotropies are related to the physical mechanism by which the solar corona and solar wind are heated [27, 41].

Near the Sun heavy ions are stronger heated than protons and electrons. These findings have strengthened the arguments in favour of the kinetic ion-cyclotron model of heating and acceleration of particles in the solar wind. However, this mechanism has a number of shortages. For example, the observed properties of low-frequency 
wave turbulence are close to those of Alfvénic modes and their power spectrum has a maximum around onetwo hours. In order to realize the ion-cyclotron resonance, Hollweg [28] assumed that low-frequency waves by the nonlinear cascade should finally turn to high-frequency modes. Within the frames of our fluid model such observed low-frequency modes can easily be explained. Indeed, in the ideal case (without a heat flux, $\gamma=0$ ) near the instability threshold of the Alfvénic fire hose modes it is possible to receive very low frequencies. For the nonideal case (with a heat flux, $\gamma \neq 0$ ) the mirror modes have every chance to explain the observable low frequencies. The new observed facts which can essentially modify our ideas about the physical nature of the solar wind are presented in a recent paper [7]. It appears that the solar wind consists of sets of magnetic filaments. We think that the nonlinear evolution of the large-scale mirror modes are capable of creating such structures.

\subsection{Identification of wave modes}

In the preceding Section 7 we presented the relations between the fluctuations of the plasma mass density and the magnetic field as well as the two components of the fluid speed. These formulae can be used for identifying the modes; in particular the simple analytic estimates of the asymptotic limiting cases are helpful for recognizing easily the modes in the observed data. Modern space and ground-based observations with spectral and imaging methods allow to detect a reach spectrum of different kinds of wave motions in the corona [2, 3, 42, 59]. From such observed data we get information mainly on the amplitudes of density perturbations and the fluid velocity component along the line-of-sight, the wave frequency, the phase speed, the mode life time, and on the spatial orientation of the magnetic loops along which the waves are running. These data allow us to compare the observed wave motions with theoretical predictions [43]. So far all interpretations are based on the well developed isotropic MHD wave theory which is based on the collision-dominated plasma description. However, in such theoretical interpretations we should be more careful, especially concerning the outer corona. For example, even in the lower corona close to the transition region the simulated electron heat flux is not described correctly by the collision-dominated Spitzer law [34]. The observed fluxes are closer to the collisionless theoretical estimates. The appearance of anisotropic wave modes is an important evidence: if there exists strong enough heat fluxes, then the phase velocities along the magnetic field will differ from those in the opposite direction. Observed life times can be compared with the growing times of the instabilities.

Our theory should be further improved. In the 16-moments transport equations the next order terms should be retained. Finite particle gyroradii should result in a $k$-dependence of the maximum of the growing instability rates such as shown in the kinetic theory [35]. An inclusion of small collisional terms in the basic equations would be the best way to describe the coronal plasma.

Acknowledgements The present work has been supported by the German Science Foundation (DFG) under grant No. 436 RUS 113/931/0-1 (R) and by the Russian Foundation for Basic Research (RFFI) under project No. 09-02-00494 which is gratefully acknowledged.

\section{References}

[1] B. W. Abraham-Shraunder, Plasma Phys. 15, 375 (1973).

[2] M. J. Aschwanden, Physics of the Solar Corona. An Introduction with Problems and Solutions (2 nd edition, Springer, Berlin-Heidelberg-New York, 2005), 892 pp.

[3] B.W. Aschwanden, in: Waves \& Oscillations in the Solar Atmosphere: Heating and Magneto-Seismology, ed. R. Erdenyi, IAU Symp. 247, 257 (2008).

[4] A. R. Barakat and R. W. Schunk, Plasma Phys. 24, 389 (1982).

[5] V. B. Baranov and K. V. Krasnobayev, Hydrodynamics of Cosmic Plasma (Nauka, Moscow, 1977), 335 pp.

[6] A. Barnes, Phys. Fluids 9, 1843 (1966).

[7] J.E. Brovsky, Phys. Rev. Letters 105, 111102 (2010).

[8] S. Chandrasekhar, A. N. Kaufman, and K. M. Watson, Proc. R. Soc. London A 245, 435 (1958).

[9] G. F. Chew, M. L. Goldberger, and F. E. Low, Proc. Roy. Soc. London A 236, 112 (1956).

[10] H. G. Demars and R. W. Schunk, J. Phys. D 12, 1051 (1979).

[11] N. S. Dzhalilov, V. D. Kuznetsov, and J. Staude, Astron. Astrophys. 489, 769 (2008).

[12] S. P. Gary, S. A. Fuselier, and B. J. Anderson, J. Geophys. Res. 98, 9181 (1993).

[13] S. P. Gary, H. Li, S. O. Rourke, and D. J. Winske, J. Geophys. Res. 103, 1467 (1998).

[14] V. Gedalin, M. Balikhin, R. L. Stangeway, and C. T. Russel, J. Geophys. Res. 107(A2), 1018 (2002). 
[15] M. Gedalin and Y.E. Lyubarsky, Phys. Plasmas 8, 2934 (2001).

[16] N. U. Grooker and G. L. Siscoe, J. Geophys. Res. 82, 185 (1977).

[17] A. Hasegawa, Phys. Fluids 12, 2642 (1969).

[18] A. Hasegawa, Plasma Instabilities and Nonlinear Effects (Springer, New York, 1975).

[19] L. N. Hau and B. U. Ö. Sonnerup, Geophys. Res. Lett. 20, 1763 (1993).

[20] L. N. Hau, J. Geophys. Res. 101, 2655 (1996).

[21] P. Hellinger and H. Matsumoto, J. Geophys. Res. 105, 10519 (2000).

[22] P. Hellinger and H. Matsumoto, J. Geophys. Res. 106, 13215 (2001).

[23] P. Hill, G. Paschmann, R. A. Treumann, W. Baumjohann, N. Sckopke, and H. Lühr, J. Geophys. Res. 100, 9575 (1995).

[24] J. V. Hollweg and H. J. Völk, J. Geophys. Res. 75, 5297 (1970).

[25] J. V. Hollweg, J. Geophys. Res. 79, 3845 (1974).

[26] J. V. Hollweg, J. Geophys. Res. 81, 1649 (1976).

[27] J. V. Hollweg and P. A. Isenberg, J. Geophys. Res. 107, 1147 (2002).

[28] J. V. Hollweg, J. Astrophys. Astr. 29, 217 (2008).

[29] Y. Kato, M. Tajiri, and T. Taniuti, J. Phys. Soc. Japan 21, 765 (1966).

[30] A. B. Kichenko and K. N. Stepanov, JETF USSR 38, 1840 (1960).

[31] M. G. Kivelson and D. J. Southwood, J. Geophys. Res. 101, 17365 (1996).

[32] R. M. Kulsrud, in: Handbook of Plasma Physics, Vol. 1, eds. A. Galeev and R. N. Sudan, North-Holland, Amsterdam, 115 (1983).

[33] V. D. Kuznetsov and V. N. Oraevskii, Astronomy Letters 18, 547 (1992).

[34] S. Landi and F. G. E. Pantellini, Astron. Astrophys. 372, 686 (2001).

[35] K. R. Lang, Astrophysical Formulae (3 rd edition, Springer, Berlin-Heidelberg-New York, 1999), 614 pp.

[36] X. Li, J. Geophys. Res. 104, 19773 (1999).

[37] X. Li and S. R. Habbal, J. Geophys. Res. 105, 27377 (2000).

[38] O. Lie-Svendsen, E. Leer, and V. H. Hansteen, J. Geophys. Res. 106, 8217 (2001).

[39] S. M. Mahajan and R. D. Hazeltine, Phys. Plasmas 9, 1882 (2002).

[40] E. Marsch and A. K. Richter, Annales Geophysicae 5 A (2), 71 (1987).

[41] E. Marsch, Living Rev. Solar Phys. 3, 1 (2006).

[42] M. S. Marsh, R. W. Walsh, and S. Plunkett, Astrophys. J. 697, 1674 (2009).

[43] V. M. Nakariakov and E. Verwichte, Living Rev. Solar Phys. 2, 3 (2005).

[44] E. L. Olsen and E. Leer, , J. Geophys. Res. 104, 9963 (1999).

[45] V. Oraevskii, R. Chodura, and W. Feneberg, Plasma Phys. 10, 819 (1968).

[46] V. N. Oraevskii, Y. V. Konikov, and G. V. Chazanov, Transport processes in anisotropic near-Earth plasma (Nauka, Moscow, 1985), 173 pp.

[47] G. Paesold and A. O. Benz, Astron. Astrophys. 351, 741 (1999).

[48] E. N. Parker, Phys. Rev. 109, 1874 (1958).

[49] K. B. Quest and V. D. Shapiro, J. Geophys. Res. 101, 24457 (1996).

[50] J. J. Ramos, Phys. Plasmas 10, 3601 (2003).

[51] A. A. Schekochihin and S. C. Cowley, Phys. Plasmas 13, 056501 (2006).

[52] P. Sharma, G. W. Hammett, E. Quataert, and J. M. Stone, Astrophys. J. 637, 952 (2006).

[53] P. B. Snyder, G. W. Hammett, and W. Dorland, Phys. Plasmas 4, 3974 (1997).

[54] D. J. Southwood and M. G. Kivelson, J. Geophys. Res. 98, 9181 (1993).

[55] M. Tajiri, J. Phys. Soc. Jpn. 22, 1482 (1967).

[56] W. B. Thompson, An Introduction to Plasma Physics (Pergamon, New York, 1964), p. 216.

[57] A. A. Vedenov and R.Z. Sagdeev, Dokl. Akad. Nauk SSSR 3, 278 (1958).

[58] B. J. Wang and L. N. Hau, J. Geophys. Res. 108 (A12), 1463 (2003).

[59] T. J. Wang, L. Ofman, and J. M. Davila, Astrophys. J. 696, 1448 (2009). 